\title{
EFFICIENT AND RELIABLE HIERARCHICAL ERROR ESTIMATES FOR THE DISCRETIZATION ERROR OF ELLIPTIC OBSTACLE PROBLEMS
}

\author{
RALF KORNHUBER AND QINGSONG ZOU
}

\begin{abstract}
We present and analyze novel hierarchical a posteriori error estimates for self-adjoint elliptic obstacle problems. Our approach differs from straightforward, but nonreliable estimators by an additional extra term accounting for the deviation of the discrete free boundary in the localization step. We prove efficiency and reliability on a saturation assumption and a regularity condition on the underlying grid. Heuristic arguments suggest that the extra term is of higher order and preserves full locality. Numerical computations confirm our theoretical findings.
\end{abstract}

\section{INTRODUCTION}

Hierarchical a posteriori error estimates are based on the extension of the given finite element space $\mathcal{S}$ by an incremental space $\mathcal{V}$. After discretization of the actual defect problem with respect to the extended space $\mathcal{S}+\mathcal{V}$, hierarchical splitting and subsequent localization give rise to local defect problems associated with lowdimensional subspaces of $\mathcal{V}$. These local subproblems can be often solved exactly providing local contributions that finally sum up to the desired a posteriori estimate of the error. We refer to the pioneering work of Zienkiewicz et al. [32] and Deuflhard et al. 10] or to the monographs of Verfürth [26] and Ainsworth \& Oden [1].

An attractive feature of hierarchical a posteriori error estimates is their robustness. For linear self-adjoint elliptic problems, local lower bounds come without unknown constants and global upper bounds (up to higher order terms) do not depend on jumps of the coefficients resolved by the underlying mesh [27, 28, 31]. The upper bound is often proved on the so-called saturation assumption that the extended space $\mathcal{S}+\mathcal{V}$ provides a more accurate approximation than the original space $\mathcal{S}$ [4, 10. The saturation assumption holds, if data oscillation is relatively small [12]. A direct proof based on local $L^{2}$-projections is contained in [33.

Another advantage of hierarchical error estimators is their intriguing simplicity. As a consequence, hierarchical concepts have been applied to various nonsmooth nonlinear problems [13, 17, in particular, to obstacle problems [14, 16, 24, 33] or two-body contact problems in linear elasticity 23. Surprisingly good effectivity

Received by the editor September 17, 2008 and, in revised form, June 22, 2009.

2010 Mathematics Subject Classification. Primary 65N15, 65N30.

The authors gratefully acknowledge substantial support by Carsten Gräser and Oliver Sander through fruitful discussions and numerical assistance. The second author is supported in part by NSFC under the grant 10601070 and in part by Alexander von Humboldt Foundation hosted by Freie Universität in Berlin.

(C)2010 American Mathematical Society Reverts to public domain 28 years from publication 
ratios were observed in all of these applications. Moreover, the local contributions resulting from the local defect problems provided effective and fully local error indicators in adaptive refinement.

In this paper, we concentrate on scalar self-adjoint elliptic obstacle problems. While residual-based error estimates [7, 20, 21, 22, 25] or averaging techniques [3] are now well understood for this problem class, the analysis of hierarchical error estimates still seems to be in its infancy. Very recently, Siebert and Veeser [24] derived efficient and reliable hierarchical error estimates for the energy functional in obstacle problems which were later improved by Zou et al. 33. On the other hand, straightforward hierarchical error estimates for the discretization error [14] might fail to provide upper bounds at all, because reliability is lost in the localization step (see, e.g., the counterexample at the end of Section 2). Related estimators [16] are more reliable but mesh-independent upper bounds are still missing as well.

Here, we present an extension of the straightforward estimator [14 by an additional extra term accounting for the deviation of the discrete free boundary in the course of the localization step. In this way, we are able to establish meshindependent lower and upper bounds for the discretization error. The proof is carried out on a saturation assumption, a discrete conforming condition, and a regularity condition on the underlying grid. More precisely, we assume that the off-diagonal elements of the stiffness matrix are nonpositive so that a monotonicity argument can be applied. Numerical computations indicate that this quite restrictive condition seems not to be necessary for mesh-independence.

The novel extra term is a sum of local residuals associated with certain exceptional nodes. The exceptional nodes always lie in the coincidence set or in its neighborhood. Hence, our a posteriori error estimates reduce to well-known results [4, 10, if no obstacle is present. Heuristic reasoning suggests that the exceptional nodes are concentrated at the discrete free boundary so that the extra term is expected to be of higher order. This explains why the straightforward estimator 14] works well in practice. Moreover, the extra term preserves full locality and there is no overestimation of the error inside of the coincidence set. Our theoretical considerations are nicely supported by numerical experiments.

Throughout this paper, " $A \lesssim B$ " means that $A$ can be bounded by $B$ multiplied with a generic constant depending only on the shape regularity of the actual triangulation $\mathcal{T}$, and " $A \sim B$ " stands for " $A \lesssim B$ " and " $B \lesssim A$ ".

\section{Hierarchical extensions AND lOCAL DEFECT PROBlems}

Let $\Omega \subset \mathbb{R}^{d}, d=2,3$, be a bounded, polygonal or polyhedral domain with Lipschitz-continuous boundary $\partial \Omega$ and denote $H=H_{0}^{1}(\Omega)$. We consider the obstacle problem

$$
u \in K: \quad a(u, v-u) \geq \ell(v-u) \quad \forall v \in K,
$$

involving the $H$-elliptic, symmetric bilinear form

$$
a(v, w)=\int_{\Omega} \nabla v \cdot \nabla w d x, \quad v, w \in H,
$$

with the associated energy norm $\|v\|=a(v, v)^{\frac{1}{2}}$, and a bounded linear functional $\ell \in H^{\prime}$. The closed, convex subset

$$
K=\{v \in H \mid v \geq \psi \text { a.e. in } \Omega\} \subset H
$$


is induced by an obstacle function $\psi \in C(\bar{\Omega})$ satisfying $\psi \leq 0$ on $\partial \Omega$. It is well known [15] that (2.1) admits a unique solution $u \in K$.

Let $\mathcal{T}$ be a conforming and shape regular triangulation of $\Omega$ with $\mathcal{N}$ and let $\mathcal{E}$ denote the set of all interior vertices and edges, respectively. We introduce the space $\mathcal{S} \subset H$ of piecewise linear finite elements on $\mathcal{T}$ spanned by the nodal basis $\left\{\phi_{P} \mid P \in \mathcal{N}\right\}$. Now the finite element discretization of (2.1) reads as

$$
u_{\mathcal{S}} \in K_{\mathcal{S}}: \quad a\left(u_{\mathcal{S}}, v-u_{\mathcal{S}}\right) \geq \ell\left(v-u_{\mathcal{S}}\right) \quad \forall v \in K_{\mathcal{S}}
$$

with discrete constraints

$$
K_{\mathcal{S}}=\{v \in \mathcal{S} \mid v(P) \geq \psi(P) \quad \forall P \in \mathcal{N}\} \subset \mathcal{S} .
$$

Note that, in general, $K_{\mathcal{S}} \not \subset K$. The discrete problem (2.3) admits a unique solution $u_{\mathcal{S}}$ which is characterized by the discrete complementarity conditions

$$
\left(\psi-u_{\mathcal{S}}\right)(P) \leq 0, \quad\left\langle\sigma_{\mathcal{S}}, \phi_{P}\right\rangle \leq 0, \quad\left(\psi-u_{\mathcal{S}}\right)(P)\left\langle\sigma_{\mathcal{S}}, \phi_{P}\right\rangle=0 \quad \forall P \in \mathcal{N}
$$

involving the discrete residual $\sigma_{\mathcal{S}} \in H^{\prime}$, defined by

$$
\left\langle\sigma_{\mathcal{S}}, v\right\rangle=\ell(v)-a\left(u_{\mathcal{S}}, v\right), \quad v \in H .
$$

The error $e=u-u_{\mathcal{S}}$ is the unique solution of the continuous defect problem

$$
e \in D: \quad a(e, v-e) \geq\left\langle\sigma_{\mathcal{S}}, v-e\right\rangle \quad \forall v \in D
$$

with defect constraints

$$
D=\left\{v \in H \mid v \geq \psi-u_{\mathcal{S}} \text { a.e. in } \Omega\right\} \subset H .
$$

In order to derive a computable approximation of $e \in H$, (2.5) is discretized by another finite element space $\mathcal{Q}$ which should be larger than $\mathcal{S}$. Following [10, 14, 16, 17, we select the space $\mathcal{Q} \subset H$ of piecewise quadratic finite elements on $\mathcal{T}$. Each function $v \in \mathcal{Q}$ is uniquely determined by its nodal values in $P \in \mathcal{N}_{\mathcal{Q}}=$ $\mathcal{N} \cup\left\{x_{E} \mid E \in \mathcal{E}\right\}$, where $x_{E}$ stands for the midpoint of $E \in \mathcal{E}$. We consider the discrete defect problem

$$
e_{\mathcal{Q}} \in D_{\mathcal{Q}}: \quad a\left(e_{\mathcal{Q}}, v-e_{\mathcal{Q}}\right) \geq\left\langle\sigma_{\mathcal{S}}, v-e_{\mathcal{Q}}\right\rangle \quad \forall v \in D_{\mathcal{Q}}
$$

with discrete defect constraints

$$
D_{\mathcal{Q}}=\left\{v \in \mathcal{Q} \mid v(P) \geq \psi(P)-u_{\mathcal{S}}(P) \quad \forall P \in \mathcal{N}_{\mathcal{Q}}\right\} .
$$

Observe that $u_{\mathcal{Q}}=u_{\mathcal{S}}+e_{\mathcal{Q}} \in \mathcal{Q}$ is just the piecewise quadratic finite element approximation which could be directly computed from

$$
u_{\mathcal{Q}} \in K_{\mathcal{Q}}: \quad a\left(u_{\mathcal{Q}}, v-u_{\mathcal{Q}}\right) \geq \ell\left(v-e_{\mathcal{Q}}\right) \quad \forall v \in K_{\mathcal{Q}}
$$

with discrete constraints

$$
K_{\mathcal{Q}}=\left\{v \in \mathcal{Q} \mid v(P) \geq \psi(P) \quad \forall P \in \mathcal{N}_{\mathcal{Q}}\right\} .
$$

It is well known [1, 2, 4, 16, 17, that the so-called saturation assumption

$$
\left\|u-u_{\mathcal{Q}}\right\| \leq \beta\left\|u-u_{\mathcal{S}}\right\|, \quad \beta<1,
$$

implies the a posteriori error estimate

$$
(1+\beta)^{-1}\left\|e_{\mathcal{Q}}\right\| \leq\left\|u-u_{\mathcal{S}}\right\| \leq(1-\beta)^{-1}\left\|e_{\mathcal{Q}}\right\| .
$$

In particular, the saturation assumption (2.8) implicitly accounts for nonconforming errors as resulting from $K_{\mathcal{S}} \not \subset K$ and $K_{\mathcal{Q}} \not \subset K$. The importance of the saturation assumption (2.8) explains the usual selection of $\mathcal{Q}$ : As the piecewise quadratic 
approximation $u_{\mathcal{Q}}$ of $u$ is of higher order for smooth data [6], the saturation assumption (2.8) can be expected to hold true for sufficiently fine meshes. Moreover, $\beta$ even tends to zero for increasing refinement so that the a posteriori error estimate $\left\|e_{\mathcal{Q}}\right\|$ is asymptotically exact for piecewise quadratics. This is not the case for an alternative selection of $\mathcal{Q}$ consisting of piecewise linear finite elements on the uniformly refined triangulation $\mathcal{T}^{\prime}$. Nevertheless, well-known optimal error estimates 8 indicate that (2.8) can still be expected to be satisfied for sufficiently fine mesh size. On the other hand, for any finite dimensional space $\mathcal{Q} \subset H$ there is a functional $\ell \in H^{\prime}$ such that the saturation assumption (2.8) is violated [4]. For unconstrained problems, small data oscillation implies the saturation assumption [12]. For obstacle problems, this kind of practical criterion for (2.8) seems to be wide open (however, see [33]). Moreover, even for smooth data, the order of piecewise quadratics is not optimal but limited by $3 / 2$. This indicates that the saturation assumption (2.8) for obstacle problems is much more restrictive than in the unconstrained case.

The evaluation of $e_{\mathcal{Q}}$ is still far too costly to be used as an a posteriori error estimator. In order to simplify the discrete defect problem (2.5), we exploit that $\mathcal{Q}$ can be regarded as a hierarchical extension of $\mathcal{S}$, i.e.,

$$
\mathcal{Q}=\mathcal{S}+\mathcal{V}, \quad \mathcal{V}=\operatorname{span}\left\{\phi_{E} \mid E \in \mathcal{E}\right\}
$$

involving the quadratic bubble functions $\phi_{E} \in \mathcal{Q}$ characterized by $\phi_{E}(P)=\delta_{x_{E}, P}$, $\forall P \in \mathcal{N}_{\mathcal{Q}}$ (Kronecker- $\delta$ ). See (3.1) for a hierarchical decomposition of quadratic nodal basis functions.

Remark 2.1. Our subsequent analysis literally carries over to hierarchical extensions as spanned by other edge bubble functions. For example, we could as well define $\phi_{E}$ as the piecewise linear nodal basis functions associated with the new vertices $x_{E} \in \mathcal{N}^{\prime} \backslash \mathcal{N}$ of the uniformly refined triangulation $\mathcal{T}^{\prime}$ or, equivalently, select $\mathcal{Q}$ to be the space of piecewise linear finite elements on $\mathcal{T}^{\prime}$.

Using the uniquely determined splitting $v=v_{\mathcal{S}}+v_{\mathcal{V}}$ and $w=w_{\mathcal{S}}+w_{\mathcal{V}}$ of $v, w \in \mathcal{Q}$ into $v_{\mathcal{S}}, w_{\mathcal{S}} \in \mathcal{S}$ and $v_{\mathcal{V}}, w_{\mathcal{V}} \in \mathcal{V}$, we define the bilinear form

$$
a_{\mathcal{Q}}\left(v_{\mathcal{Q}}, w_{\mathcal{Q}}\right)=a\left(v_{\mathcal{S}}, w_{\mathcal{S}}\right)+\sum_{E \in \mathcal{E}} v_{\mathcal{V}}\left(x_{E}\right) w_{\mathcal{V}}\left(x_{E}\right) a\left(\phi_{E}, \phi_{E}\right)
$$

and the associated energy norm $\|v\|_{\mathcal{Q}}=a_{\mathcal{Q}}(v, v)^{\frac{1}{2}}$ on $\mathcal{Q}$. Note that $a_{\mathcal{Q}}(\cdot, \cdot)$ is just a two-level version of the well-known hierarchical basis preconditioner [27, 28, 30, 31]. It is a result of decoupling $\mathcal{S}$ and $\mathcal{V}$ and further diagonalization on $\mathcal{V}$. The norm equivalence

$$
a_{\mathcal{Q}}(v, v) \sim a(v, v) \quad \forall v \in \mathcal{Q}
$$

follows from the estimates

$$
\left\|v_{\mathcal{S}}\right\|+\left\|v_{\mathcal{V}}\right\| \sim\|v\|, \quad\left\|v_{\mathcal{V}}\right\|_{\mathcal{Q}}=\left(\sum_{E \in \mathcal{E}} v_{\mathcal{V}}\left(x_{E}\right)^{2} a\left(\phi_{E}, \phi_{E}\right)\right)^{\frac{1}{2}} \sim\left\|v_{\mathcal{V}}\right\|,
$$

as obtained from related local versions [4, 10,

$$
\left\|v_{\mathcal{S}}\right\|_{T}+\left\|v_{\mathcal{V}}\right\|_{T} \sim\|v\|_{T}, \quad\left(\sum_{E \in \mathcal{E}_{T}} v_{\mathcal{V}}\left(x_{E}\right)^{2} a\left(\phi_{E}, \phi_{E}\right)\right)^{\frac{1}{2}} \sim\left\|v_{\mathcal{V}}\right\|_{T},
$$


where $\mathcal{E}_{T}$ denotes the set of edges of $T \in \mathcal{T}$.

It has been shown in [16] that the unique solution $\varepsilon_{\mathcal{Q}}$ of the associated preconditioned defect problem

$$
\varepsilon_{\mathcal{Q}} \in D_{\mathcal{Q}}: \quad a_{\mathcal{Q}}\left(\varepsilon_{\mathcal{Q}}, v-\varepsilon_{\mathcal{Q}}\right) \geq\left\langle\sigma_{\mathcal{S}}, v-\varepsilon_{\mathcal{Q}}\right\rangle \quad \forall v \in D_{\mathcal{Q}}
$$

inherits the norm equivalence (2.12), i.e.,

$$
\left\|\varepsilon_{\mathcal{Q}}\right\|_{\mathcal{Q}} \sim\left\|e_{\mathcal{Q}}\right\| .
$$

Due to the remaining coupling of $\mathcal{S}$ and $\mathcal{V}$ by the constraints $D_{\mathcal{Q}}$, the unique solution $\varepsilon_{\mathcal{Q}}$ is still not available in closed form. Hence, we introduce the subset

$$
D_{\mathcal{V}}=\left\{v \in \mathcal{V} \mid v\left(x_{E}\right) \geq \psi\left(x_{E}\right)-u_{\mathcal{S}}\left(x_{E}\right) \forall E \in \mathcal{E}\right\} \subset D_{\mathcal{Q}}
$$

and the corresponding approximate discrete defect problem

$$
\tilde{\varepsilon}_{\mathcal{V}} \in D_{\mathcal{V}}: \quad a_{\mathcal{Q}}\left(\tilde{\varepsilon}_{\mathcal{V}}, v-\tilde{\varepsilon}_{\mathcal{V}}\right) \geq\left\langle\sigma_{\mathcal{S}}, v-\tilde{\varepsilon}_{\mathcal{V}}\right\rangle \quad \forall v \in D_{\mathcal{V}} .
$$

The solution $\tilde{\varepsilon_{\mathcal{V}}} \in \mathcal{V}$ of (2.17) is explicitly given by

$$
\tilde{\varepsilon}_{\mathcal{V}}\left(x_{E}\right)=\left\{\begin{aligned}
-d_{E}\left\|\phi_{E}\right\|^{-1} & \forall E \in \mathcal{E}_{1}=\left\{E \in \mathcal{E} \mid \rho_{E} \leq-d_{E}\right\}, \\
\rho_{E}\left\|\phi_{E}\right\|^{-1} & \forall E \in \mathcal{E}_{2}=\left\{E \in \mathcal{E} \mid \rho_{E}>-d_{E}\right\},
\end{aligned}\right.
$$

where we have set

$$
d_{E}=\left(u_{\mathcal{S}}\left(x_{E}\right)-\psi\left(x_{E}\right)\right)\left\|\phi_{E}\right\|, \quad \rho_{E}=\left\langle\sigma_{\mathcal{S}}, \phi_{E}\right\rangle\left\|\phi_{E}\right\|^{-1}, \quad E \in \mathcal{E} .
$$

From a heuristic point of view, the midpoints $x_{E}$ of $E \in \mathcal{E}_{1}$ can be regarded as approximate coincidence nodes, because $\left(u_{\mathcal{S}}+\tilde{\varepsilon}_{\mathcal{V}}\right)\left(x_{E}\right)=\psi\left(x_{E}\right)$. Accordingly, the midpoints $x_{E}$ of $E \in \mathcal{E}_{2}$ can be regarded as approximate noncoincidence nodes.

The resulting straightforward a posteriori estimate

$$
\left\|\tilde{\varepsilon}_{\mathcal{V}}\right\|_{\mathcal{Q}}^{2}=\sum_{E \in \mathcal{E}} \eta_{E}^{2}, \quad \eta_{E}=\left|\tilde{\varepsilon}_{\mathcal{V}}\left(x_{E}\right)\right|\left\|\phi_{E}\right\|, \quad E \in \mathcal{E},
$$

for the discretization error $\left\|u-u_{\mathcal{S}}\right\|^{2}$ has been suggested in [14. The local contributions $\eta_{E}$ have also been used successfully as refinement indicators. In the unconstrained case, we get $\varepsilon_{\mathcal{Q}}=\tilde{\varepsilon}_{\mathcal{V}}$ so that, by (2.9) and (2.16) the error estimate (2.20) is efficient and reliable on the saturation assumption (2.8). However, this is no longer true for obstacle problems. The following counterexample shows that, in general, $\left\|\varepsilon_{\mathcal{Q}}\right\|_{\mathcal{Q}}$ is not bounded by $\left\|\tilde{\varepsilon}_{\mathcal{V}}\right\|_{\mathcal{Q}}$ at all.

Let $\Omega=(0,1), a(v, w)=\int_{0}^{1} v^{\prime} w^{\prime} d x, \psi=0$, and

$$
\ell(v)=\int_{0}^{\frac{1}{4}}(-3) v(x) d x+\int_{\frac{1}{4}}^{\frac{3}{4}} v(x) d x+\int_{\frac{3}{4}}^{1}(-3) v(x) d x .
$$

The piecewise linear finite element approximation resulting from $\mathcal{S}=\operatorname{span}\left\{\phi_{P}\right\}$, $P=\frac{1}{2}$, is $u_{\mathcal{S}}=0$, because $\ell\left(\phi_{P}\right)=0$. The corresponding piecewise quadratic finite element approximation $e_{\mathcal{Q}}$ of the error $u-u_{\mathcal{S}}$ cannot be zero, because $\left\langle\sigma_{\mathcal{S}}, \phi_{P}^{\mathcal{Q}}\right\rangle=$ $\ell\left(\phi_{P}^{\mathcal{Q}}\right)-a\left(0, \phi_{P}^{\mathcal{Q}}\right)>0$ holds for the quadratic nodal basis function $\phi_{P}^{\mathcal{Q}}$. On the other hand, we compute $\left\langle\sigma_{\mathcal{S}}, \phi_{E}\right\rangle<0$ for $E=[1,1 / 2]$ and $E=[1 / 2,1]$ so that $\tilde{\varepsilon}_{\mathcal{V}}=0$.

One might conclude that the hierarchical error estimate (2.20) needs some extension accounting for the deviation from $\left\|\varepsilon_{\mathcal{Q}}\right\|_{\mathcal{Q}}$ to $\left\|\tilde{\varepsilon}_{\mathcal{V}}\right\|_{\mathcal{Q}}$ in the localization step from (2.15) to (2.17). This will be the subject of the following section. 


\section{EFFICIENCY AND RELIABILITY}

For each $P \in \mathcal{N}$ and $E \in \mathcal{E}$, we define

$$
\omega_{P}=\operatorname{supp} \phi_{P}, \quad \gamma_{P}=\left\{E^{\prime} \in \mathcal{E} \mid P \in E^{\prime}\right\}, \quad \omega_{E}=\operatorname{supp} \phi_{E} .
$$

We will make use of the hierarchical representation

$$
\phi_{P}^{\mathcal{Q}}=\phi_{P}-\sum_{E \in \gamma_{P}} \phi_{P}\left(x_{E}\right) \phi_{E}
$$

of the piecewise quadratic nodal basis function $\phi_{P}^{\mathcal{Q}} \in \mathcal{Q}$ associated with $P$. We further introduce the subset of exceptional nodes

$$
\mathcal{N}_{b}=\left\{P \in \mathcal{N} \mid \rho_{P}>0\right\} \subset \mathcal{N},
$$

denoting

$$
\rho_{P}=\left\langle\sigma_{\mathcal{S}}, \tilde{\phi}_{P}\right\rangle\left\|\phi_{P}\right\|^{-1}, \quad \tilde{\phi}_{P}=\phi_{P}-\sum_{E \in \gamma_{P}^{1}} \phi_{P}\left(x_{E}\right) \phi_{E}, \quad \gamma_{P}^{1}=\gamma_{P} \cap \mathcal{E}_{1} .
$$

Now we are ready to state the main result of this paper.

Theorem 3.1. Assume that the saturation assumption (2.8) is satisfied, that the discrete conforming condition

$$
u_{\mathcal{S}} \in K_{\mathcal{Q}}
$$

holds, and that $\mathcal{T}$ satisfies the regularity condition

$$
a\left(\phi_{P}, \phi_{P^{\prime}}\right) \leq 0 \quad \forall P, P^{\prime} \in \mathcal{N}, P \neq P^{\prime} .
$$

Then the hierarchical error estimator (2.20) is efficient and reliable in the sense that

$$
\left\|u-u_{\mathcal{S}}\right\|^{2} \sim \sum_{E \in \mathcal{E}} \eta_{E}^{2}+\sum_{P \in \mathcal{N}_{b}} \rho_{P}^{2}
$$

holds with $\eta_{E}$ and $\rho_{P}$ defined in (2.20) and (3.2), respectively.

According to condition (3.3) there is no nonconforming error in the piecewise linear approximation $u_{\mathcal{S}}$ of $u_{\mathcal{Q}}$. By definition, (3.3) can be reformulated as $0 \in D_{\mathcal{Q}}$ or, equivalently, $d_{E} \geq 0$ (cf. (2.19) $)$. This property will be used in the proofs of Lemma 3.5 and Proposition 3.6 below. It is difficult to check the discrete conforming condition (3.3) a priori. However, it can be guaranteed by additional assumptions on $\psi$, for example, that $\psi$ is convex along the edges of $\mathcal{T}$.

It is well known that the regularity condition (3.4) is satisfied in $d=2$ space dimensions, if (and only if with some possible rare exceptions near the boundary) $\mathcal{T}$ is a Delaunay triangulation 9. Xu and Zikatanov 29] extended this characterization to arbitrary space dimensions. It is sufficient in $d=2$ or $d=3$ dimensions that $\mathcal{T}$ consists of nonobtuse triangles or tetrahedra, respectively, [18. A tetrahedron is called nonobtuse, if all of its six dihedral angles between faces are nonobtuse. It is not easy to preserve nonobtuse angles and thus the regularity condition (3.4) under adaptive refinement. In $d=2$ dimensions the classical red/green refinement strategy usually destroys the Delaunay property: Red refinement preserves interior angles but green closure does not. Longest edge bisection preserves nonobtuse triangles only in very special cases such as, e.g., the initial partition of the unit square into four congruent subtriangles. Nonobtuse adaptive refinement is even harder in $d=3$ space dimensions. We refer to Korotov et al. [18, 19] for further information. 
Remark 3.2. The local contributions $\eta_{E}$ and $\rho_{P}$ to the a posteriori error estimate (3.5) are explicitly computable from (2.20) and (3.2). Moreover, in light of the following heuristic arguments, the additional term $\sum_{P \in \mathcal{N}_{b}} \rho_{P}^{2}$ can be expected to be of higher order.

Assume that no coincidence occurs in the neighboring midpoints $x_{E}$ of $P$ in the sense that $E \in \mathcal{E}_{2}$ holds for all $E \in \gamma_{P}$ or, equivalently, $\gamma_{P}^{1}=\emptyset$. Then $\tilde{\phi}_{P}=\phi_{P}$. Therefore, complementarity (2.4) implies $\left\langle\sigma_{\mathcal{S}}, \tilde{\phi}_{P}\right\rangle=\left\langle\sigma_{\mathcal{S}}, \phi_{P}\right\rangle \leq 0$ and thus $P \notin \mathcal{N}_{b}$. Conversely, assume that coincidence takes place in all neighboring midpoints $x_{E}$ of $P$ in the sense that $E \in \mathcal{E}_{1}$ holds for all $E \in \gamma_{P}$ or, equivalently, $\gamma_{P}^{1}=\gamma_{P}$. Then $\tilde{\phi}_{P}=\phi_{P}$. Similar to $\sigma_{\mathcal{S}}$, the discrete quadratic residual $\sigma_{\mathcal{Q}}=\ell-a\left(u_{\mathcal{Q}}, \cdot\right)$ satisfies $\left\langle\sigma_{\mathcal{Q}}, \tilde{\phi}_{P}\right\rangle=\left\langle\sigma_{\mathcal{Q}}, \phi_{P}^{\mathcal{Q}}\right\rangle \leq 0$. For nondegenerate problems, even inequality holds, if $u_{\mathcal{Q}}(P)=\psi(P)$. In this case, we can expect $\left\langle\sigma_{\mathcal{S}}, \tilde{\phi}_{P}\right\rangle \leq 0$ and thus $P \notin \mathcal{N}_{b}$, if $\left\langle\sigma_{\mathcal{Q}}-\sigma_{\mathcal{S}}, \phi_{P}^{\mathcal{Q}}\right\rangle \leq\left\|u_{\mathcal{Q}}-u_{\mathcal{S}}\right\|\left\|\phi_{P}^{\mathcal{Q}}\right\|$ is small enough.

As a consequence of the above heuristic reasoning, the set of exceptional nodes $\mathcal{N}_{b}$ can be expected to concentrate along the continuous free boundary, if the mesh size $h$ is sufficiently small. In addition, we have $\rho_{P}^{2}=\mathcal{O}\left(h^{d+2}\right)$, if $P$ is close to the free boundary. Hence, we can expect $\sum_{P \in \mathcal{N}_{b}} \rho_{P}^{2}=\mathcal{O}\left(h^{3}\right)$, i.e. that the additional term is of higher order.

The remainder of this section is devoted to the proof of Theorem 3.1. In light of the basic error estimate (2.9), the equivalence (2.16), and the representation (2.20), it is sufficient to show

$$
\left\|\varepsilon_{\mathcal{Q}}\right\|_{\mathcal{Q}}^{2} \sim\left\|\tilde{\varepsilon_{\mathcal{V}}}\right\|_{\mathcal{Q}}^{2}+\sum_{P \in \mathcal{N}_{b}} \rho_{P}^{2}
$$

We start to prove (3.6) by collecting some local properties of the solution $\varepsilon_{\mathcal{Q}}$ of the preconditioned defect problem (2.15).

Lemma 3.3. The inequality $\varepsilon_{\mathcal{Q}}\left(x_{E}\right)>\psi\left(x_{E}\right)-u_{\mathcal{S}}\left(x_{E}\right)$ implies

$$
a_{\mathcal{Q}}\left(\varepsilon_{\mathcal{Q}}, \phi_{E}\right)=\left\langle\sigma_{\mathcal{S}}, \phi_{E}\right\rangle \text {. }
$$

Let $\varepsilon_{\mathcal{Q}}=\varepsilon_{\mathcal{S}}+\varepsilon_{\mathcal{V}}$ denote the hierarchical splitting of $\varepsilon_{\mathcal{Q}}$ into $\varepsilon_{\mathcal{S}} \in \mathcal{S}$ and $\varepsilon_{\mathcal{V}} \in \mathcal{V}$. Then

$$
\varepsilon_{\mathcal{V}}\left(x_{E}\right)=\max \left\{\rho_{E}\left\|\phi_{E}\right\|^{-1}, \psi\left(x_{E}\right)-\left(u_{\mathcal{S}}+\varepsilon_{\mathcal{S}}\right)\left(x_{E}\right)\right\}
$$

holds for all $E \in \mathcal{E}$.

Proof. Inserting $v=\varepsilon_{\mathcal{Q}}+\phi_{E} \in \mathcal{D}_{\mathcal{Q}}$ into (2.15), we get

$$
a_{\mathcal{Q}}\left(\varepsilon_{\mathcal{Q}}, \phi_{E}\right) \geq\left\langle\sigma_{\mathcal{S}}, \phi_{E}\right\rangle, \quad \forall E \in \mathcal{E}
$$

If $\varepsilon_{\mathcal{Q}}\left(x_{E}\right)>\psi\left(x_{E}\right)-u_{\mathcal{S}}\left(x_{E}\right)$, then there is a sufficiently small $\alpha>0$ such that $\varepsilon_{\mathcal{Q}}\left(x_{E}\right)-\alpha \phi_{E}\left(x_{E}\right) \geq \psi\left(x_{E}\right)-u_{\mathcal{S}}\left(x_{E}\right)$. Inserting $v=\left(\varepsilon_{\mathcal{Q}}\left(x_{E}\right)-\alpha \phi_{E}\left(x_{E}\right)\right) \phi_{E} \in \mathcal{D}_{\mathcal{Q}}$ into (2.15), we get the converse inequality

$$
a_{\mathcal{Q}}\left(\varepsilon_{\mathcal{Q}},-\alpha \phi_{E}\right) \geq\left\langle\sigma_{\mathcal{S}},-\alpha \phi_{E}\right\rangle .
$$

In order to show (3.8), we start with the splitting $\varepsilon_{\mathcal{Q}}=\varepsilon_{\mathcal{S}}+\varepsilon_{\mathcal{V}}$. By definition of $a_{\mathcal{Q}}(\cdot, \cdot)$ and $\rho_{E}$ in (2.11) and (2.19), respectively, (3.9) can be rewritten as

$$
\varepsilon_{\mathcal{V}}\left(x_{E}\right) \geq \rho_{E}\left\|\phi_{E}\right\|^{-1} \text {. }
$$

We have just shown that equality holds, if $\varepsilon_{\mathcal{Q}}\left(x_{E}\right)>\psi\left(x_{E}\right)-u_{\mathcal{S}}\left(x_{E}\right)$ or, equivalently, $\varepsilon_{\mathcal{V}}\left(x_{E}\right)>\psi\left(x_{E}\right)-\left(u_{\mathcal{S}}+\varepsilon_{\mathcal{S}}\right)\left(x_{E}\right)$. This concludes the proof. 
Note that the inequality $\varepsilon_{\mathcal{Q}}(P)>\psi(P)-u_{\mathcal{S}}(P)$ does not imply

$$
a_{\mathcal{Q}}\left(\varepsilon_{\mathcal{Q}}, \phi_{P}\right)=\left\langle\sigma_{\mathcal{S}}, \phi_{P}\right\rangle \text {. }
$$

Indeed, we have $\varepsilon_{\mathcal{Q}}-\alpha \phi_{P} \notin \mathcal{D}_{\mathcal{Q}}$ for all $\alpha>0$, if $\varepsilon_{\mathcal{Q}}\left(x_{E}\right)=\psi\left(x_{E}\right)-u_{\mathcal{S}}\left(x_{E}\right)$ holds for some $E \in \gamma_{P}$.

In the next two lemmata we further analyze the components $\varepsilon_{\mathcal{S}} \in \mathcal{S}$ and $\varepsilon_{\mathcal{V}} \in \mathcal{V}$ of the hierarchical splitting $\varepsilon_{\mathcal{Q}}=\varepsilon_{\mathcal{S}}+\varepsilon_{\mathcal{V}} \in \mathcal{D}_{\mathcal{Q}}$.

Lemma 3.4. Assume that the regularity condition (3.4) is satisfied. Then

$$
\varepsilon_{\mathcal{S}} \geq 0
$$

Proof. We decompose $\varepsilon_{\mathcal{S}}=\varepsilon_{\mathcal{S}}^{+}+\varepsilon_{\mathcal{S}}^{-}$into its positive part $\varepsilon_{\mathcal{S}}^{+} \in \mathcal{S}$ and its negative part $e_{\mathcal{S}}^{-} \in \mathcal{S}$ with the nodal values

$$
\varepsilon_{\mathcal{S}}^{+}(P)=\max \left(0, \varepsilon_{\mathcal{S}}(P)\right), \quad \varepsilon_{\mathcal{S}}^{-}(P)=\min \left(0, \varepsilon_{\mathcal{S}}(P)\right), \quad P \in \mathcal{N} .
$$

It is sufficient to show $\varepsilon_{\mathcal{S}}^{-}=0$. Inserting $v=\varepsilon_{\mathcal{Q}}+\phi_{P} \in \mathcal{D}_{\mathcal{Q}}$ into (2.15) we get

$$
a\left(\varepsilon_{\mathcal{S}}, \phi_{P}\right)=a_{\mathcal{Q}}\left(\varepsilon_{\mathcal{Q}}, \phi_{P}\right) \geq\left\langle\sigma_{\mathcal{S}}, \phi_{P}\right\rangle \quad \forall P \in \mathcal{N}
$$

so that $\varepsilon_{\mathcal{S}}^{-}(P) \leq 0$ yields

$$
a\left(\varepsilon_{\mathcal{S}}, \varepsilon_{\mathcal{S}}^{-}\right) \leq\left\langle\sigma_{\mathcal{S}}, \varepsilon_{\mathcal{S}}^{-}\right\rangle
$$

According to (2.4), $u_{\mathcal{S}}(P)>\psi(P)$ implies $\left\langle\sigma_{\mathcal{S}}, \phi_{P}\right\rangle=0$, and $u_{\mathcal{S}}(P)=\psi(P)$ leads to $\varepsilon_{\mathcal{S}}^{-}(P)=0$. Thus we have shown $e_{\mathcal{S}}^{-}(P)\left\langle\sigma_{\mathcal{S}}, \phi_{P}\right\rangle=0$ for all $P \in \mathcal{N}$ and therefore

$$
a\left(\varepsilon_{\mathcal{S}}, \varepsilon_{\mathcal{S}}^{-}\right) \leq\left\langle\sigma_{\mathcal{S}}, \varepsilon_{\mathcal{S}}^{-}\right\rangle=\sum_{P \in \mathcal{N}} e_{\mathcal{S}}^{-}(P)\left\langle\sigma_{\mathcal{S}}, \phi_{P}\right\rangle=0
$$

Utilizing the regularity condition (3.4), i.e., $a\left(\phi_{P_{1}}, \phi_{P_{2}}\right) \leq 0$ for $P_{1} \neq P_{2}$ and the identity $\varepsilon_{\mathcal{S}}^{+}\left(P_{1}\right) \varepsilon_{\mathcal{S}}^{-}\left(P_{2}\right)=0$ for $P_{1}=P_{2}$, we obtain

$$
-a\left(\varepsilon_{\mathcal{S}}^{+}, \varepsilon_{\mathcal{S}}^{-}\right)=\sum_{P_{1}, P_{2} \in \mathcal{N}} \varepsilon_{\mathcal{S}}^{+}\left(P_{1}\right)\left(-\varepsilon_{\mathcal{S}}^{-}\left(P_{2}\right)\right) a\left(\phi_{P_{1}}, \phi_{P_{2}}\right) \leq 0 .
$$

The above two estimates yield

$$
a\left(\varepsilon_{\mathcal{S}}^{-}, \varepsilon_{\mathcal{S}}^{-}\right)=a\left(\varepsilon_{\mathcal{S}}, \varepsilon_{\mathcal{S}}^{-}\right)-a\left(\varepsilon_{\mathcal{S}}^{+}, \varepsilon_{\mathcal{S}}^{-}\right) \leq 0 .
$$

This concludes the proof.

As a direct consequence of the preceding two lemmata, we can now compare the piecewise quadratic components $\varepsilon_{\mathcal{V}}$ and $\tilde{\varepsilon}_{\mathcal{V}}$.

Lemma 3.5. Assume that the discrete conforming condition (3.3) and the regularity condition (3.4) are satisfied. Then

$$
\rho_{E}\left\|\phi_{E}\right\|^{-1} \leq \varepsilon_{\mathcal{V}}\left(x_{E}\right) \leq \tilde{\varepsilon}_{\mathcal{V}}\left(x_{E}\right) \quad \forall E \in \mathcal{E} .
$$

Moreover, $\tilde{\varepsilon}_{\mathcal{V}}\left(x_{E}\right) \leq 0$ holds for all $E \in \mathcal{E}_{1}$ and both inequalities in (3.11) hold with equality for all $E \in \mathcal{E}_{2}$.

Proof. From Lemma 3.3 it is known that

$$
\varepsilon_{\mathcal{V}}\left(x_{E}\right)=\max \left\{\rho_{E}\left\|\phi_{E}\right\|^{-1}, \psi\left(x_{E}\right)-\left(u_{\mathcal{S}}+\varepsilon_{\mathcal{S}}\right)\left(x_{E}\right)\right\}
$$

while

$$
\tilde{\varepsilon}_{\mathcal{V}}\left(x_{E}\right)=\max \left\{\rho_{E}\left\|\phi_{E}\right\|^{-1}, \psi\left(x_{E}\right)-u_{\mathcal{S}}\left(x_{E}\right)\right\}
$$

holds by (2.18). Now (3.11) follows from Lemma 3.4. The remaining assertions are obtained from the representation (2.18) of $\tilde{\varepsilon}_{\mathcal{V}}\left(x_{E}\right)$ and the conforming condition (3.3). 
In light of Lemma 3.5, $\mathcal{E}_{2}$ can be regarded as a subset of the piecewise quadratic approximation of noncoincidence nodes in the sense that

$$
\varepsilon_{\mathcal{Q}}\left(x_{E}\right)>\psi\left(x_{E}\right)-u_{\mathcal{S}}\left(x_{E}\right) \quad \forall E \in \mathcal{E}_{2} .
$$

On the other hand, $E \in \mathcal{E}_{1}$ does not necessarily imply $\varepsilon_{Q}\left(x_{E}\right)=\psi\left(x_{E}\right)-u_{\mathcal{S}}\left(x_{E}\right)$.

We are now ready to prove the efficiency of our hierarchical error estimator.

Proposition 3.6. Assume that the discrete conforming condition (3.3) and the regularity condition (3.4) are satisfied. Then the following estimate holds:

$$
\left\|\tilde{\varepsilon}_{\mathcal{V}}\right\|_{\mathcal{Q}}^{2}+\sum_{P \in \mathcal{N}_{b}} \rho_{P}^{2} \lesssim\left\|\varepsilon_{\mathcal{Q}}\right\|_{\mathcal{Q}}^{2}
$$

Proof. By Lemma 3.5, we have

$$
\left|\tilde{\mathcal{E}}_{\mathcal{V}}\left(x_{E}\right)\right| \leq\left|\varepsilon_{\mathcal{V}}\left(x_{E}\right)\right| \quad \forall E \in \mathcal{E}
$$

and therefore

$$
\left\|\tilde{\varepsilon}_{\mathcal{V}}\right\|_{\mathcal{Q}}^{2} \leq\left\|\varepsilon_{\mathcal{V}}\right\|_{\mathcal{Q}}^{2} \leq\left\|\varepsilon_{\mathcal{Q}}\right\|_{\mathcal{Q}}^{2}
$$

It remains to show that $\sum_{P \in \mathcal{N}_{b}} \rho_{P}^{2} \lesssim\left\|\varepsilon_{\mathcal{Q}}\right\|_{Q}^{2}$. Let $P \in \mathcal{N}_{b}$. Note that $\tilde{\phi}_{P}=$ $\phi_{P}^{\mathcal{Q}}+\sum_{E \in \gamma_{P} \cap \mathcal{E}_{2}} \phi_{P}\left(x_{E}\right) \phi_{E}$. Inserting $\varepsilon_{\mathcal{Q}}+\phi_{P}^{\mathcal{Q}} \in \mathcal{D}_{\mathcal{Q}}$ into (2.15), we get

$$
\left\langle\sigma_{\mathcal{S}}, \phi_{P}^{\mathcal{Q}}\right\rangle \leq a_{\mathcal{Q}}\left(\varepsilon_{\mathcal{Q}}, \phi_{P}^{\mathcal{Q}}\right)
$$

which, in combination with (3.12) and (3.7), leads to

$$
\left\langle\sigma_{\mathcal{S}}, \tilde{\phi}_{P}\right\rangle=\left\langle\sigma_{\mathcal{S}}, \phi_{P}^{\mathcal{Q}}\right\rangle+\sum_{E \in \gamma_{P} \cap \mathcal{E}_{2}} \phi_{P}\left(x_{E}\right)\left\langle\sigma_{\mathcal{S}}, \phi_{E}\right\rangle \leq a_{\mathcal{Q}}\left(\varepsilon_{\mathcal{Q}}, \tilde{\phi}_{P}\right) .
$$

Now we insert the definitions of $a_{\mathcal{Q}}(\cdot, \cdot)$ and $\tilde{\phi}_{P}$ to obtain

$$
\begin{aligned}
0<\left\langle\sigma_{\mathcal{S}}, \tilde{\phi}_{P}\right\rangle & \leq a\left(\varepsilon_{\mathcal{S}}, \phi_{P}\right)-\sum_{E \in \gamma_{P}^{1}} \phi_{P}\left(x_{E}\right) \varepsilon_{\mathcal{V}}\left(x_{E}\right) a\left(\phi_{E}, \phi_{E}\right) \\
& \leq\left\|\varepsilon_{\mathcal{S}}\right\|_{\omega_{P}}\left\|\phi_{P}\right\|+\sum_{E \in \gamma_{P}^{1}} \mid \varepsilon_{\mathcal{V}}\left(x_{E}\right)\left\|\phi_{E}\right\|^{2}
\end{aligned}
$$

exploiting the Cauchy-Schwarz inequality, the triangle inequality and $\left|\phi_{P}\left(x_{E}\right)\right| \leq 1$. Another application of the Cauchy-Schwarz inequality provides that

$$
\rho_{P}^{2} \lesssim\left\|\varepsilon_{\mathcal{S}}\right\|_{\omega_{P}}^{2}+\sum_{E \in \gamma_{P}^{1}}\left|\varepsilon_{\mathcal{V}}\left(x_{E}\right)\right|^{2}\left\|\phi_{E}\right\|^{2} .
$$

We sum up this estimate for all $P \in \mathcal{N}_{b}$ and exploit shape regularity, to get

$$
\begin{aligned}
\sum_{P \in \mathcal{N}_{b}} \rho_{P}^{2} & \lesssim \sum_{P \in \mathcal{N}_{b}}\left\|\varepsilon_{\mathcal{S}}\right\|_{\omega_{P}}^{2}+\sum_{P \in \mathcal{N}_{b}} \sum_{E \in \gamma_{P}^{1}}\left|\varepsilon_{\mathcal{V}}\left(x_{E}\right)\right|^{2}\left\|\phi_{E}\right\|^{2} \\
& \lesssim\left\|\varepsilon_{\mathcal{S}}\right\|^{2}+\left\|\varepsilon_{\mathcal{V}}\right\|_{\mathcal{Q}}^{2}=\left\|\varepsilon_{\mathcal{Q}}\right\|_{\mathcal{Q}}^{2} .
\end{aligned}
$$

This concludes the proof.

In preparation of proving reliability, we state two further lemmata.

Lemma 3.7. The inequality $\varepsilon_{\mathcal{Q}}(P)>\psi(P)-u_{\mathcal{S}}(P)$ implies

$$
a_{\mathcal{Q}}\left(\varepsilon_{\mathcal{Q}}, \phi_{P}^{\mathcal{Q}}\right)=\left\langle\sigma_{\mathcal{S}}, \phi_{P}^{\mathcal{Q}}\right\rangle, \quad a_{\mathcal{Q}}\left(\varepsilon_{\mathcal{Q}}, \tilde{\phi}_{P}\right)=\left\langle\sigma_{\mathcal{S}}, \tilde{\phi}_{P}\right\rangle
$$

with $\phi_{P}^{\mathcal{Q}}$ and $\tilde{\phi}_{P}$ defined in (3.1) and (3.2), respectively. 
Proof. As $\phi_{P}^{\mathcal{Q}}\left(P^{\prime}\right)=\delta_{P, P^{\prime}}$ for all $P, P^{\prime} \in \mathcal{N}_{\mathcal{Q}}$, the left equality in (3.14) can be shown in a similar way as (3.7). Exploiting (3.12)

$$
\begin{aligned}
a_{\mathcal{Q}}\left(\varepsilon_{\mathcal{Q}}, \tilde{\phi}_{P}\right) & =a_{\mathcal{Q}}\left(\varepsilon_{\mathcal{Q}}, \phi_{P}^{\mathcal{Q}}\right)+\sum_{E \in \gamma_{P} \cap \mathcal{E}_{2}} \phi_{P}\left(x_{E}\right) a_{\mathcal{Q}}\left(\varepsilon_{\mathcal{Q}}, \phi_{E}\right) \\
& =\left\langle\sigma_{\mathcal{S}}, \phi_{P}^{\mathcal{Q}}\right\rangle+\sum_{E \in \gamma_{P} \cap \mathcal{E}_{2}} \phi_{P}\left(x_{E}\right)\left\langle\sigma_{\mathcal{S}}, \phi_{E}\right\rangle=\left\langle\sigma_{\mathcal{S}}, \tilde{\phi}_{P}\right\rangle
\end{aligned}
$$

then follows from the left equality in (3.14) and (3.7).

Lemma 3.8. The estimate

$$
\left|v(P)-v\left(x_{E}\right)\right| \lesssim\|v\|_{\omega_{P}}\left\|\phi_{P}\right\|^{-1}
$$

holds for all $P \in \mathcal{N}, E \in \gamma_{P}$ and $v \in \mathcal{Q}$.

Proof. Let $P \in \mathcal{N}, E \in \gamma_{P}$ and $v=v_{\mathcal{S}}+v_{\mathcal{V}} \in \mathcal{Q}$ with $v_{\mathcal{S}} \in \mathcal{S}$ and $v_{\mathcal{V}} \in \mathcal{V}$. Since

$$
v\left(x_{E}\right)=v_{\mathcal{V}}\left(x_{E}\right)+v_{\mathcal{S}}\left(x_{E}\right)=v_{\mathcal{V}}\left(x_{E}\right)+\sum_{P^{\prime} \in \mathcal{N}} v_{\mathcal{S}}\left(P^{\prime}\right) \phi_{P^{\prime}}\left(x_{E}\right),
$$

and $\sum_{P^{\prime} \in \mathcal{N}} \phi_{P^{\prime}}\left(x_{E}\right)=1$, it is clear that

$$
v\left(x_{E}\right)-v(P)=v_{\mathcal{V}}\left(x_{E}\right)+\sum_{P^{\prime} \in \mathcal{N}, P^{\prime} \neq P}\left(v_{\mathcal{S}}\left(P^{\prime}\right)-v_{\mathcal{S}}(P)\right) \phi_{P^{\prime}}\left(x_{E}\right) .
$$

Note that $\phi_{P^{\prime}}\left(x_{E}\right) \neq 0$, if and only if $P^{\prime} \in \omega_{P}$. Select $T \in \mathcal{T}$ such that $P, P^{\prime} \in T \subset$ $\omega_{P}$ and let $h_{T}=\operatorname{diam} T$. Then shape regularity implies

$$
\left|v_{\mathcal{S}}\left(P^{\prime}\right)-v_{\mathcal{S}}(P)\right| \leq h_{T}\left|\nabla v_{\mathcal{S}}\right|_{T} \mid \lesssim h_{T}^{1-d / 2}\left\|v_{\mathcal{S}}\right\|_{\omega_{P}},
$$

because $\left.\nabla v_{\mathcal{S}}\right|_{T}$ is constant. Utilizing again shape regularity, we get $\left\|\phi_{P}\right\| \sim h_{T}^{d / 2-1}$ and therefore

$$
\left|v_{\mathcal{S}}\left(P^{\prime}\right)-v_{\mathcal{S}}(P)\right| \lesssim\left\|\phi_{P}\right\|^{-1}\left\|v_{\mathcal{S}}\right\|_{\omega_{P}}
$$

Now choose $T \in \mathcal{T}$ such that $x_{E} \in T \subset \omega_{P}$. Then

$$
\left|v_{\mathcal{V}}\left(x_{E}\right)\right| \leq\left(\sum_{E^{\prime} \in \mathcal{E}_{T}} v_{\mathcal{V}}\left(x_{E^{\prime}}\right)^{2}\right)^{\frac{1}{2}} \lesssim h_{T}^{1-d / 2}\left\|v_{\mathcal{V}}\right\|_{T} \lesssim\left\|\phi_{P}\right\|^{-1}\left\|v_{\mathcal{V}}\right\|_{\omega_{P}}
$$

follows from $\left\|\phi_{E^{\prime}}\right\| \sim h_{T}^{d / 2-1}$ and the equivalence (2.14) of local norms. Inserting these estimates into (3.16), we get

$$
\left|v\left(x_{E}\right)-v(P)\right| \lesssim\left(\left\|v_{\mathcal{V}}\right\|_{\omega_{P}}+\left\|v_{\mathcal{S}}\right\|_{\omega_{P}}\right)\left\|\phi_{P}\right\|^{-1} .
$$

Now the assertion follows from the left estimate in (2.14) and the shape regularity of $\mathcal{T}$.

We are now ready to prove the reliability of our hierarchical error estimator.

Proposition 3.9. Assume that the discrete conforming condition (3.3) and the regularity condition (3.4) are satisfied. Then the following estimate holds:

$$
\left\|\varepsilon_{\mathcal{Q}}\right\|_{\mathcal{Q}}^{2} \lesssim\left\|\tilde{\varepsilon}_{\mathcal{V}}\right\|_{\mathcal{Q}}^{2}+\sum_{P \in \mathcal{N}_{b}} \rho_{P}^{2}
$$


Proof. Using the splitting $\varepsilon_{\mathcal{Q}}=\varepsilon_{\mathcal{S}}+\varepsilon_{\mathcal{V}}$, we obtain

$$
a_{\mathcal{Q}}\left(\varepsilon_{\mathcal{Q}}, \varepsilon_{\mathcal{Q}}\right)=a_{\mathcal{Q}}\left(\varepsilon_{\mathcal{Q}}, \varepsilon_{\mathcal{S}}\right)+\sum_{E \in \mathcal{E}} \varepsilon_{\mathcal{V}}\left(x_{E}\right) a_{\mathcal{Q}}\left(\varepsilon_{\mathcal{Q}}, \phi_{E}\right)
$$

by definition (2.11) of $a_{\mathcal{Q}}(\cdot, \cdot)$. As a consequence of $a_{\mathcal{Q}}\left(\varepsilon_{\mathcal{Q}}, \phi_{E}\right)=\varepsilon_{\mathcal{V}}\left(x_{E}\right)\left\|\phi_{E}\right\|^{2}$ and Lemma 3.5 this leads to

$$
a_{\mathcal{Q}}\left(\varepsilon_{\mathcal{Q}}, \varepsilon_{\mathcal{Q}}\right)=a_{\mathcal{Q}}\left(\varepsilon_{\mathcal{Q}}, \varepsilon_{\mathcal{S}}\right)+\sum_{E \in \mathcal{E}_{1}} \varepsilon_{\mathcal{V}}\left(x_{E}\right) a_{\mathcal{Q}}\left(\varepsilon_{\mathcal{Q}}, \phi_{E}\right)+\sum_{E \in \mathcal{E}_{2}} \rho_{E}^{2}
$$

Utilizing the splitting $\mathcal{E}_{1}=\mathcal{E}_{1}^{+} \cup \mathcal{E}_{1}^{-}$,

$$
\mathcal{E}_{1}^{+}=\left\{E \in \mathcal{E}_{1} \mid \varepsilon_{\mathcal{Q}}\left(x_{E}\right)>0\right\}, \quad \mathcal{E}_{1}^{-}=\left\{E \in \mathcal{E}_{1} \mid \varepsilon_{\mathcal{Q}}\left(x_{E}\right) \leq 0\right\},
$$

and $\varepsilon_{\mathcal{V}}\left(x_{E}\right)=\varepsilon_{\mathcal{Q}}\left(x_{E}\right)-\varepsilon_{\mathcal{S}}\left(x_{E}\right)$, we rewrite this identity according to

$$
a_{\mathcal{Q}}\left(\varepsilon_{\mathcal{Q}}, \varepsilon_{\mathcal{Q}}\right)=I_{1}+I_{2}+I_{3}+I_{4}
$$

where

$$
I_{1}=a_{\mathcal{Q}}\left(\varepsilon_{\mathcal{Q}}, \varepsilon_{\mathcal{S}}\right)-\sum_{E \in \mathcal{E}_{1}} \varepsilon_{\mathcal{S}}\left(x_{E}\right) a_{\mathcal{Q}}\left(\varepsilon_{\mathcal{Q}}, \phi_{E}\right)
$$

and

$$
I_{2}=\sum_{E \in \mathcal{E}_{1}^{+}} \varepsilon_{\mathcal{Q}}\left(x_{E}\right) a_{\mathcal{Q}}\left(\varepsilon_{\mathcal{Q}}, \phi_{E}\right), \quad I_{3}=\sum_{E \in \mathcal{E}_{1}^{-}} \varepsilon_{\mathcal{Q}}\left(x_{E}\right) a_{\mathcal{Q}}\left(\varepsilon_{\mathcal{Q}}, \phi_{E}\right), \quad I_{4}=\sum_{E \in \mathcal{E}_{2}} \rho_{E}^{2} .
$$

We will treat these four terms in the given order.

In the first step, we consider the terms $I_{1}$ and $I_{2}$. We start with the first expression $a_{\mathcal{Q}}\left(\varepsilon_{\mathcal{Q}}, \varepsilon_{\mathcal{S}}\right)$ occurring in $I_{1}$. Let

$$
\mathcal{N}_{1}=\left\{P \in \mathcal{N} \mid \gamma_{P}^{1} \neq \emptyset\right\}
$$

Note that $\mathcal{N}_{b} \subset \mathcal{N}_{1}$, because $P \in \mathcal{N} \backslash \mathcal{N}_{1}$ implies $\tilde{\phi}_{P}=\phi_{P}$ and thus $P \in \mathcal{N} \backslash \mathcal{N}_{b}$. Let us consider some $P \in \mathcal{N} \backslash \mathcal{N}_{1}$ and assume that $\varepsilon_{\mathcal{Q}}(P)=\varepsilon_{\mathcal{S}}(P)>0 \geq \psi(P)-u_{\mathcal{S}}(P)$. As $\tilde{\phi}_{P}=\phi_{P}$, Lemma 3.7 provides

$$
a_{\mathcal{Q}}\left(\varepsilon_{\mathcal{Q}}, \phi_{P}\right)=\left\langle\sigma_{\mathcal{S}}, \phi_{P}\right\rangle \leq 0 .
$$

As $\varepsilon_{\mathcal{S}}(P)<0$ does not occur (cf. Lemma 3.4), we have shown

$$
a_{\mathcal{Q}}\left(\varepsilon_{\mathcal{Q}}, \varepsilon_{\mathcal{S}}\right)=\sum_{P \in \mathcal{N}} \varepsilon_{\mathcal{S}}(P) a_{\mathcal{Q}}\left(\varepsilon_{\mathcal{Q}}, \phi_{P}\right) \leq \sum_{P \in \mathcal{N}_{1}} \varepsilon_{\mathcal{S}}(P) a_{\mathcal{Q}}\left(\varepsilon_{\mathcal{Q}}, \phi_{P}\right)
$$

Let us consider the second expression occurring in $I_{1}$. We insert the nodal representation $\varepsilon_{\mathcal{S}}\left(x_{E}\right)=\sum_{P \in \mathcal{N}_{E}} \varepsilon_{\mathcal{S}}(P) \phi_{P}\left(x_{E}\right)$ with $\mathcal{N}_{E}=\left\{P \in \mathcal{N} \mid \phi_{P}\left(x_{E}\right) \neq 0\right\}$ and rearrange terms to obtain

$$
\begin{aligned}
\sum_{E \in \mathcal{E}_{1}} \varepsilon_{\mathcal{S}}\left(x_{E}\right) a_{\mathcal{Q}}\left(\varepsilon_{\mathcal{Q}}, \phi_{E}\right) & =\sum_{E \in \mathcal{E}_{1}}\left(\sum_{P \in \mathcal{N}_{E}} \varepsilon_{\mathcal{S}}(P) \phi_{P}\left(x_{E}\right)\right) a_{\mathcal{Q}}\left(\varepsilon_{\mathcal{Q}}, \phi_{E}\right) \\
& =\sum_{P \in \mathcal{N}_{1}} \varepsilon_{\mathcal{S}}(P) a_{\mathcal{Q}}\left(\varepsilon_{\mathcal{Q}}, \sum_{E \in \gamma_{P}^{1}} \phi_{P}\left(x_{E}\right) \phi_{E}\right) .
\end{aligned}
$$

Combining (3.19) and (3.20), we have shown

$$
I_{1} \leq \sum_{P \in \mathcal{N}_{1}} \varepsilon_{\mathcal{S}}(P) a_{\mathcal{Q}}\left(\varepsilon_{\mathcal{Q}}, \tilde{\phi}_{P}\right) .
$$

Now Lemma 3.7 yields

$$
a_{\mathcal{Q}}\left(\varepsilon_{\mathcal{Q}}, \tilde{\phi}_{P}\right)=\left\langle\sigma_{\mathcal{S}}, \tilde{\phi}_{P}\right\rangle
$$


for all $P \in \mathcal{N}_{1} \subset \mathcal{N}$ satisfying $\varepsilon_{\mathcal{Q}}(P)=\varepsilon_{\mathcal{S}}(P)>0 \geq \psi(P)-u_{\mathcal{S}}(P)$. By Lemma 3.4 the case $\varepsilon_{\mathcal{S}}(P)<0$ does not occur. Hence, by the definition of $\mathcal{N}_{b} \subset \mathcal{N}_{1}$, we have

$$
I_{1} \leq \sum_{P \in \mathcal{N}_{1}} \varepsilon_{\mathcal{S}}(P) a_{\mathcal{Q}}\left(\varepsilon_{\mathcal{Q}}, \tilde{\phi}_{P}\right)=\sum_{P \in \mathcal{N}_{b}} \varepsilon_{\mathcal{S}}(P)\left\langle\sigma_{\mathcal{S}}, \tilde{\phi}_{P}\right\rangle .
$$

Now let $P \in \mathcal{N}_{b}$. As $\mathcal{N}_{b} \subset \mathcal{N}_{1}$, it is clear that $\gamma_{P}^{1} \neq \emptyset$. Hence, there exists $E_{P} \in \gamma_{P}^{1}$ with the property

$$
\varepsilon_{\mathcal{Q}}\left(x_{E_{P}}\right)=\min \left\{\varepsilon_{\mathcal{Q}}\left(x_{E}\right) \mid E \in \gamma_{P}^{1}\right\} .
$$

By Lemma 3.8, we have

$$
\left|\varepsilon_{\mathcal{Q}}(P)-\varepsilon_{\mathcal{Q}}\left(x_{E_{P}}\right)\right| \lesssim\left\|\varepsilon_{\mathcal{Q}}\right\|_{\omega_{P}}\left\|\phi_{P}\right\|^{-1} .
$$

Either $E_{P} \in \mathcal{E}_{1}^{-}$leads to

$$
\begin{aligned}
\varepsilon_{\mathcal{S}}(P)=\varepsilon_{\mathcal{Q}}(P) & \leq \varepsilon_{\mathcal{Q}}(P)-\varepsilon_{Q}\left(x_{E_{P}}\right) \\
& \lesssim\left\|\varepsilon_{\mathcal{Q}}\right\|_{\omega_{P}}\left\|\phi_{P}\right\|^{-1}
\end{aligned}
$$

or $E_{P} \in \mathcal{E}_{1}^{+}$provides

$$
\begin{aligned}
\varepsilon_{\mathcal{S}}(P)=\varepsilon_{\mathcal{Q}}(P) & =\varepsilon_{\mathcal{Q}}(P)-\varepsilon_{Q}\left(x_{E_{P}}\right)+\varepsilon_{Q}\left(x_{E_{P}}\right) \\
& \lesssim\left\|\varepsilon_{\mathcal{Q}}\right\|_{\omega_{P}}\left\|\phi_{P}\right\|^{-1}+\varepsilon_{Q}\left(x_{E_{P}}\right) .
\end{aligned}
$$

These two inequalities can be rewritten as

$$
\varepsilon_{\mathcal{S}}(P) \leq\left\|\varepsilon_{\mathcal{Q}}\right\|_{\omega_{P}}\left\|\phi_{P}\right\|^{-1}+\max \left\{0, \varepsilon_{Q}\left(x_{E_{P}}\right)\right\} .
$$

We insert this estimate into (3.21) and apply the Cauchy-Schwarz inequality to obtain

$$
\begin{aligned}
I_{1} & \leq \sum_{P \in \mathcal{N}_{b}}\left\|\varepsilon_{\mathcal{Q}}\right\|_{\omega_{P}} \rho_{P}+\sum_{P \in \mathcal{N}_{b}} \max \left\{0, \varepsilon_{Q}\left(x_{E_{P}}\right)\right\}\left\langle\sigma_{\mathcal{S}}, \tilde{\phi}_{P}\right\rangle \\
& \lesssim\left\|\varepsilon_{\mathcal{Q}}\right\|\left(\sum_{P \in \mathcal{N}_{b}} \rho_{P}^{2}\right)^{\frac{1}{2}}+\sum_{P \in \mathcal{N}_{b}, E_{P} \in \mathcal{E}_{1}^{+}} \varepsilon_{Q}\left(x_{E_{P}}\right)\left\langle\sigma_{\mathcal{S}}, \tilde{\phi}_{P}\right\rangle .
\end{aligned}
$$

We now concentrate on the second term in (3.22). Let $E_{P} \in \mathcal{E}_{1}^{+}$. Then $\gamma_{P}^{1} \subset \mathcal{E}_{1}^{+}$. Exploiting the definition of $E_{P}$ and the conforming condition (3.3), we obtain

$$
\varepsilon_{\mathcal{Q}}\left(x_{E}\right) \geq \varepsilon_{\mathcal{Q}}\left(x_{E_{P}}\right)>0 \geq \psi\left(x_{E}\right)-u_{\mathcal{S}}\left(x_{E}\right) \quad \forall E \in \mathcal{E}_{1}^{+} .
$$

Hence, Lemma 3.3 provides

$$
a_{\mathcal{Q}}\left(\varepsilon_{Q}, \phi_{E}\right)=\left\langle\sigma_{\mathcal{S}}, \phi_{E}\right\rangle \quad \forall E \in \mathcal{E}_{1}^{+} .
$$

Utilizing $\left\langle\sigma_{\mathcal{S}}, \phi_{P}\right\rangle \leq 0$ from (2.4) and that $\left\langle\sigma_{\mathcal{S}}, \phi_{E}\right\rangle \leq 0$ holds for all $E \in \mathcal{E}_{1}$ by definition (2.18) of $\mathcal{E}_{1}$ and $d_{E} \geq 0$ (cf. (3.3) ), we now obtain

$$
\begin{aligned}
\varepsilon_{\mathcal{Q}}\left(x_{E_{P}}\right)\left\langle\sigma_{\mathcal{S}}, \tilde{\phi}_{P}\right\rangle & \leq \varepsilon_{Q}\left(x_{E_{P}}\right)\left\langle\sigma_{\mathcal{S}},-\sum_{E \in \gamma_{P}^{1}} \phi_{P}\left(x_{E}\right) \phi_{E}\right\rangle \\
& \leq \sum_{E \in \mathcal{E}_{1}^{+} \cap \gamma_{P}^{1}} \varepsilon_{\mathcal{Q}}\left(x_{E}\right)\left\langle\sigma_{\mathcal{S}},-\phi_{P}\left(x_{E}\right) \phi_{E}\right\rangle \\
& =\sum_{E \in \mathcal{E}_{1}^{+} \cap \gamma_{P}^{1}} \varepsilon_{\mathcal{Q}}\left(x_{E}\right) a_{\mathcal{Q}}\left(\varepsilon_{Q},-\phi_{P}\left(x_{E}\right) \phi_{E}\right) .
\end{aligned}
$$


We have used Lemma 3.3 in the last step. Summation over $P \in \mathcal{N}_{b}$, rearranging terms, and $\phi_{P}\left(x_{E}\right) \leq 1$ leads to

$$
\begin{aligned}
\sum_{P \in \mathcal{N}_{b}, E_{P} \in \mathcal{E}_{1}^{+}} \varepsilon_{\mathcal{Q}}\left(x_{E_{P}}\right)\left\langle\sigma_{\mathcal{S}}, \tilde{\phi}_{P}\right\rangle & \leq \sum_{P \in \mathcal{N}_{b}} \sum_{E \in \mathcal{E}_{1}^{+} \cap \gamma_{P}^{1}} \phi_{P}\left(x_{E}\right) \varepsilon_{\mathcal{Q}}\left(x_{E}\right) a_{\mathcal{Q}}\left(\varepsilon_{Q},-\phi_{E}\right) \\
& =\sum_{E \in \mathcal{E}_{1}^{+}}\left(\sum_{P \in \mathcal{N}_{b} \cap \mathcal{N}_{E}} \phi_{P}\left(x_{E}\right)\right) \varepsilon_{\mathcal{Q}}\left(x_{E}\right) a_{\mathcal{Q}}\left(\varepsilon_{Q},-\phi_{E}\right) \\
& \leq-\sum_{E \in \mathcal{E}_{1}^{+}} \varepsilon_{\mathcal{Q}}\left(x_{E}\right) a_{\mathcal{Q}}\left(\varepsilon_{Q}, \phi_{E}\right)=-I_{2} .
\end{aligned}
$$

In light of (3.22) and the norm equivalence (2.12), we have shown

$$
I_{1}+I_{2} \lesssim\left(\sum_{P \in \mathcal{N}_{b}} \rho_{P}^{2}\right)^{\frac{1}{2}}\left\|\varepsilon_{\mathcal{Q}}\right\|_{\mathcal{Q}}
$$

In the second step, we consider $I_{3}$. Exploiting (3.3), $\left|\varepsilon_{\mathcal{Q}}\left(x_{E}\right)\right|=-\varepsilon_{\mathcal{Q}}\left(x_{E}\right) \leq$ $u_{\mathcal{S}}\left(x_{E}\right)-\psi\left(x_{E}\right)=d_{E}\left\|\phi_{E}\right\|^{-1}$ holds for all $E \in \mathcal{E}_{1}^{-}$. Hence, the identity $a_{\mathcal{Q}}\left(\varepsilon_{\mathcal{Q}}, \phi_{E}\right)$ $=\varepsilon_{\mathcal{V}}\left(x_{E}\right)\left\|\phi_{E}\right\|^{2}$, the definition (2.20) of $\eta_{E}$, the Cauchy-Schwarz inequality, and the estimate $\left\|\varepsilon_{\mathcal{V}}\right\|_{\mathcal{Q}} \leq\left(\left\|\varepsilon_{\mathcal{V}}\right\|_{\mathcal{Q}}^{2}+\left\|\varepsilon_{\mathcal{S}}\right\|^{2}\right)^{1 / 2}=\left\|\varepsilon_{\mathcal{Q}}\right\|_{\mathcal{Q}}$ yield

$$
\begin{aligned}
I_{3}=\sum_{E \in \mathcal{E}_{1}^{-}} \varepsilon_{\mathcal{Q}}\left(x_{E}\right) a_{\mathcal{Q}}\left(\varepsilon_{\mathcal{Q}}, \phi_{E}\right) & \leq \sum_{E \in \mathcal{E}_{1}^{-}} d_{E}\left|\varepsilon_{\mathcal{V}}\left(x_{E}\right)\right|\left\|\phi_{E}\right\| \\
& \leq\left(\sum_{E \in \mathcal{E}_{1}} \eta_{E}^{2}\right)^{\frac{1}{2}}\left\|\varepsilon_{\mathcal{Q}}\right\|_{\mathcal{Q}} .
\end{aligned}
$$

In the third step, we consider $I_{4}$. According to Lemma 3.5. $\varepsilon_{\mathcal{V}}\left(x_{E}\right)=\tilde{\varepsilon}_{\mathcal{V}}\left(x_{E}\right)=$ $\rho_{E}\left\|\phi_{E}\right\|^{-1}$ holds for all $E \in \mathcal{E}_{2}$. Hence, the definition (2.20) of $\eta_{E}$, the CauchySchwarz inequality and the estimate $\left\|\varepsilon_{\mathcal{V}}\right\|_{\mathcal{Q}} \leq\left\|\varepsilon_{\mathcal{Q}}\right\|_{\mathcal{Q}}$ yield

$$
I_{4}=\sum_{E \in \mathcal{E}_{2}} \rho_{E}^{2}=\sum_{E \in \mathcal{E}_{2}} \eta_{E}\left|\varepsilon_{\mathcal{V}}\left(x_{E}\right)\right|\left\|\phi_{E}\right\| \leq\left(\sum_{E \in \mathcal{E}_{2}} \eta_{E}^{2}\right)^{\frac{1}{2}}\left\|\varepsilon_{\mathcal{Q}}\right\|_{\mathcal{Q}} .
$$

To conclude the proof, we insert the estimates (3.24), (3.25), and (3.26) into the representation (3.18) and obtain

$$
\left\|\varepsilon_{\mathcal{Q}}\right\|_{\mathcal{Q}} \lesssim\left(\sum_{P \in \mathcal{N}_{b}} \rho_{P}^{2}\right)^{\frac{1}{2}}+\left(\sum_{E \in \mathcal{E}_{1}} \eta_{E}^{2}\right)^{\frac{1}{2}}+\left(\sum_{E \in \mathcal{E}_{2}} \eta_{E}^{2}\right)^{\frac{1}{2}}
$$

Now the assertion follows from the Cauchy-Schwarz inequality and the representation (2.20) of $\left\|\tilde{\varepsilon}_{\mathcal{V}}\right\|_{\mathcal{Q}}$.

\section{Numerical RESUlts}

In our numerical experiments, we will consider sequences of triangulations $\mathcal{T}_{j}$, $j=0,1, \ldots, J$, as resulting from $j$ local refinement steps of an initial triangulation $\mathcal{T}_{0}$. The subscript $j$ will always refer to the corresponding triangulation $\mathcal{T}_{j}$ as, for example, in $\mathcal{N}_{j}, \mathcal{E}_{j}, \mathcal{S}_{j}, u_{\mathcal{S}_{j}}$, and so on. We either apply uniform refinement, i.e., we 
connect the midpoints of all edges $E \in \mathcal{E}_{j}$, or we apply local adaptive refinement based on the local contributions $\eta_{E}^{2}, \rho_{P}^{2}$ to the hierarchical error estimator

$$
\eta_{j}^{2}=\sum_{E \in \mathcal{E}_{j}} \eta_{E}^{2}+\rho_{j}^{2}, \quad \rho_{j}^{2}=\sum_{P \in \mathcal{N}_{j, b}} \rho_{P}^{2}
$$

as introduced in Theorem 3.1. Here, we use a variant of the following refinement strategy suggested by Dörfler [11. First, the local contributions $\eta_{E}^{2}, \rho_{P}^{2}$ are ordered according to their size. Then, proceeding from the largest to smaller contributions, we collect all entries from this list until they sum up to $(1-\theta)^{2} \eta_{j}^{2}$. Finally, if $\eta_{E}^{2}$ or $\rho_{P}^{2}$ are contained in this collection, then all triangles in the support of $\phi_{E}$ or $\phi_{P}$ are marked for refinement. Like Dörfler [11, we select $\theta=0.2$ in our computations. Note that, in general, this strategy does not preserve symmetry, because only the first of more than one entry with equal size might be collected for refinement.

4.1. Constant obstacle. Following Nochetto et al. [21, we consider the constant obstacle $\psi \equiv 0$, the domain $\Omega=(0,1)^{2}$, and the radially symmetric right-hand side

$$
\ell(v)=\int_{\Omega} f v d x, \quad f(x)= \begin{cases}-4\left(2|x|^{2}+2\left(|x|^{2}-r^{2}\right)\right), & |x|>r, \\ -8 r^{2}\left(1-\left(|x|^{2}-r^{2}\right)\right), & |x| \leq r,\end{cases}
$$

providing the radially symmetric exact solution

$$
u(x)=\left(\max \left\{|x|^{2}-r^{2}, 0\right\}\right)^{2}
$$

with corresponding boundary conditions. Like Nochetto et al. 21, we select $r=0.7$ in our numerical computations. In our first experiment, the triangulations $\mathcal{T}_{j}$, $j=1, \ldots, 9$, are obtained by uniform refinement of an initial triangulation $\mathcal{T}_{0}$ consisting of four congruent triangles.
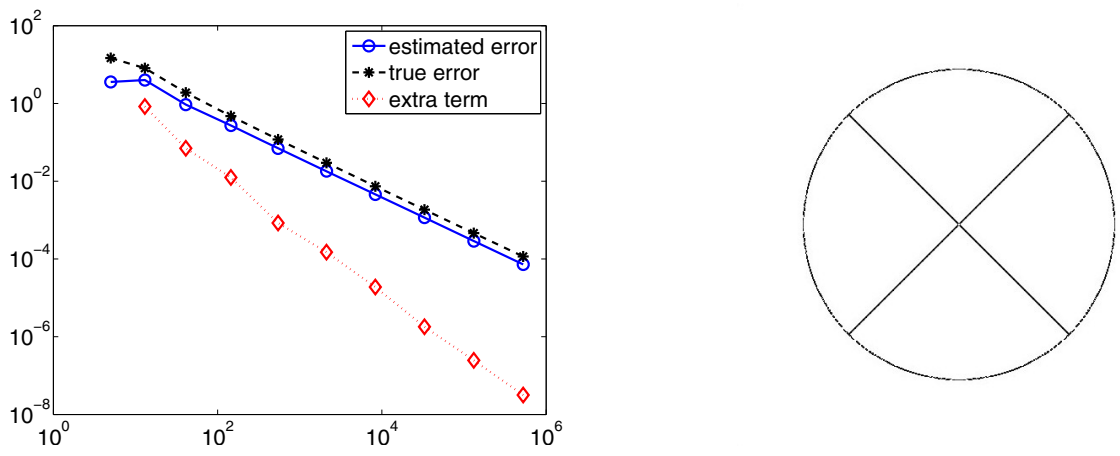

FiguRE 1. Comparison of the squared error $\left\|u-u_{\mathcal{S}_{j}}\right\|^{2}$ with the hierarchical estimator $\eta_{j}^{2}$, and distribution of the exceptional nodes $\mathcal{N}_{9, b}$.

The left picture in Figure 1 shows the squared discretization error $\left\|u-u_{\mathcal{S}_{j}}\right\|^{2}$, the hierarchical estimator $\eta_{j}^{2}$, and the extra term $\rho_{j}^{2}$ over the number of unknowns $n_{j}$. The true error seems to be approximated reasonably well. More precisely, for $j=1, \ldots, 9$ the effectivity ratios $\left\|u-u_{\mathcal{S}_{j}}\right\|^{2} / \eta_{j}^{2}$ are ranging from 0.63 to 0.79 and seem to saturate at 0.79 . This behavior is in perfect agreement with saturation (2.8) and preconditioning (2.12). Like the squared error, the estimator $\eta_{j}^{2}$ is proportional 
to $n_{j}^{-1}=\mathcal{O}\left(h_{j}^{2}\right)$ with $h_{j}$ denoting the mesh size of $\mathcal{T}_{j}$. Moreover, we observe $\rho_{j}^{2}=\mathcal{O}\left(n_{j}^{-3 / 2}\right)=\mathcal{O}\left(h_{j}^{3}\right)$, i.e., the extra term $\rho_{j}$ is of higher order, as predicted in Remark 3.2

On the other hand, the distribution of exceptional nodes $\mathcal{N}_{b}$, as illustrated in the right picture in Figure 1, is partly surprising at first. A subset of the exceptional nodes is concentrated at the circular free boundary of $u_{\mathcal{S}}$ which is supporting the heuristic reasoning in the discussion of Theorem [3.1. However, there is another subset of exceptional nodes located along the diagonals which seems to contradict our expectation that there are no exceptional nodes inside the coincidence set. The reason is that quadratic finite elements might generate spurious discrete free boundaries. Indeed, for exceptional points $P$ located inside the coincidence set, i.e., with $u_{\mathcal{S}}=\psi=0$ on $\omega_{P}$, we have $\tilde{\phi}_{P}=\phi_{P}^{\mathcal{Q}}$ and therefore

$$
0<\rho_{P}\left\|\phi_{P}\right\|=\left\langle\sigma_{\mathcal{S}}, \phi_{P}^{\mathcal{Q}}\right\rangle=\ell\left(\phi_{P}^{\mathcal{Q}}\right)=\int_{\Omega} f \phi_{P}^{\mathcal{Q}} d x .
$$

As a consequence, $u_{\mathcal{Q}}$ cannot be zero on $\omega_{P}$, because (4.27) would contradict (2.7) otherwise. Hence, there must be a spurious free boundary along the diagonals. As contributions from exceptional nodes account for the deviation of $\tilde{\varepsilon}_{\mathcal{V}}$ from the piecewise quadratic approximation $e_{\mathcal{Q}}=u_{\mathcal{Q}}-u_{\mathcal{S}}$ of the error and not from the true error $u-u_{\mathcal{S}}$, it is clear that the exceptional nodes $\mathcal{N}_{b}$ cluster along the spurious free boundary as well. This is exactly what we observe. Note that the spurious contributions $\rho_{P}^{2}$ at the diagonals are several magnitudes smaller than the others.

The reason why estimates of the form (4.27) and thus spurious free boundaries might occur is that quadratic nodal basis functions $\phi_{P}^{\mathcal{Q}}$ could be negative. This is not the case for nodal basis functions of piecewise linear finite elements on a uniformly refined triangulation $\mathcal{T}^{\prime}$. Hence, the unexpected behavior of $\mathcal{N}_{b}$ observed in Figure 1 can be avoided by replacing the quadratic bubble functions by piecewise linear ones. We emphasize that all of our theoretical considerations including efficiency and reliability literally carry over to this case (cf. Remark 2.1).
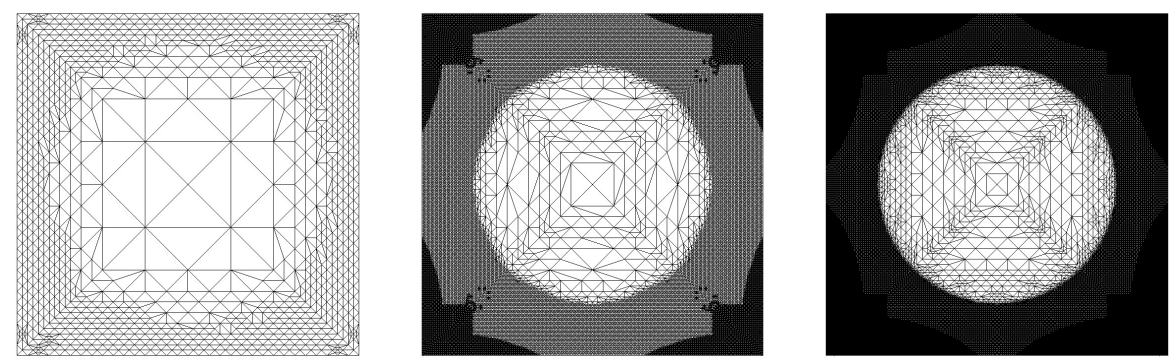

FiguRE 2. Adaptively refined triangulations $\mathcal{T}_{6}, \mathcal{T}_{10}, \mathcal{T}_{12}$.

In order to illustrate the locality of the hierarchical error estimator $\eta_{j}$, Figure 2 shows the triangulations $\mathcal{T}_{j}, j=6,10,12$, as a result of the adaptive refinement strategy described above. Note that the quadratic instability hardly influences the refinement process, because the corresponding local contributions are very small. However, effects of quadratic instability become slightly visible with increasing 
refinement. Though the adaptively refined triangulations no longer fulfill the regularity condition (3.4), we observe that the effectivity ratios $\left\|u-u_{\mathcal{S}_{j}}\right\|^{2} / \eta_{j}^{2}$ are still quite reasonable, ranging from 0.63 to 0.82 , and that the extra term $\rho_{j}$ is still of higher order.

4.2. Lipschitz obstacle. Following Nochetto et al. 21 again, we consider the domain $\Omega=\left\{x \in \mathbb{R}^{2}|| x_{1}|+| x_{2} \mid<1\right\}$, the right-hand side $\ell(v)=-5 \int_{\Omega} v(x) d x$, the Lipschitz obstacle

$$
\psi(x)=\operatorname{dist}(x, \partial \Omega)-\frac{1}{5},
$$

and homogeneous Dirichlet boundary conditions. The triangulations $\mathcal{T}_{j}, j=1,2$, $\ldots, 12$, are resulting from local adaptive refinement of the initial triangulation $\mathcal{T}_{0}$ consisting of four congruent triangles.

The final approximate solution $u_{12}$ is depicted in the left picture in Figure 3 while the right picture shows the corresponding free boundary. Observe the cusps approximated by "antennae" of sole edges. Note that this effect can be regarded as a lack of regularity of the discrete coincidence set 5 .
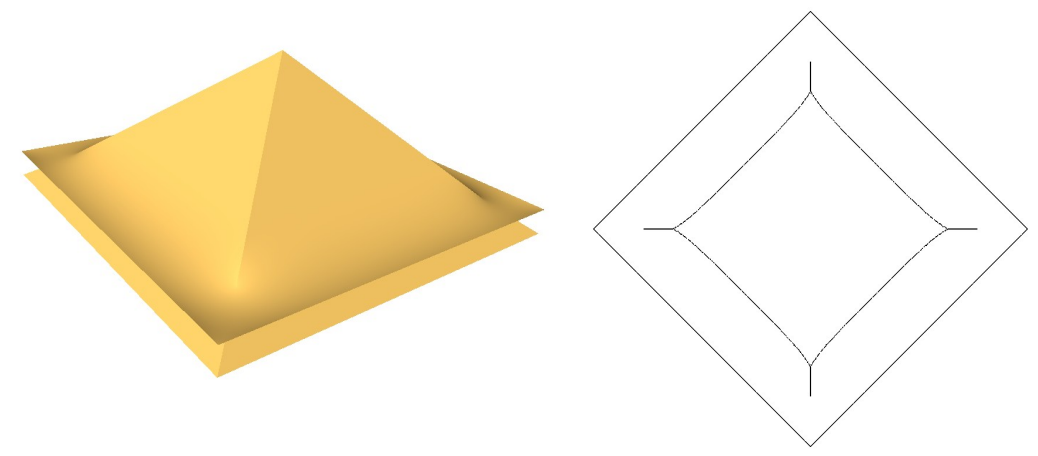

FiguRE 3. Approximate solution $u_{12}$ with obstacle function $\psi$ and associated free boundary
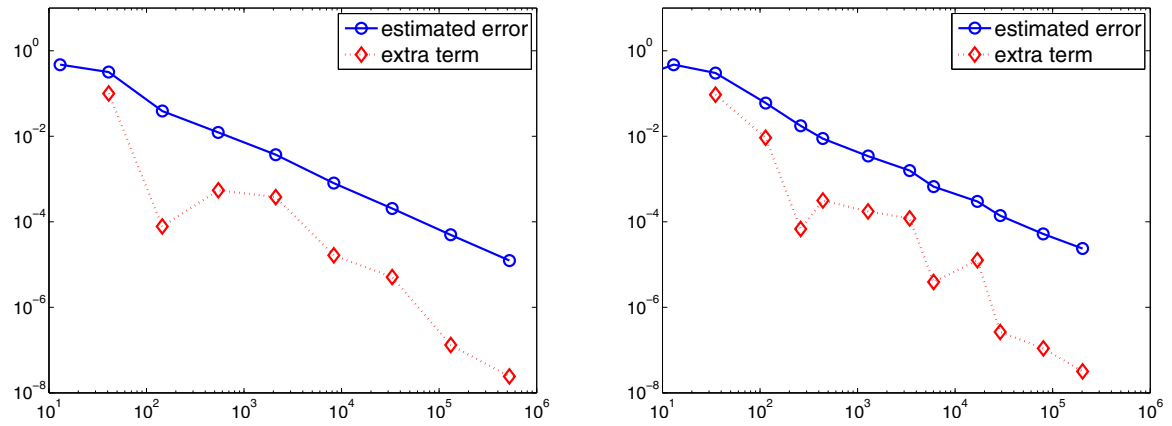

Figure 4 . The hierarchical estimator $\eta_{j}^{2}$ over the number of unknowns $n_{j}$ for the initial triangulation $\mathcal{T}_{1}$ resolving the singularities of $\psi$ : Uniform refinement (left) and adaptive refinement (right). 

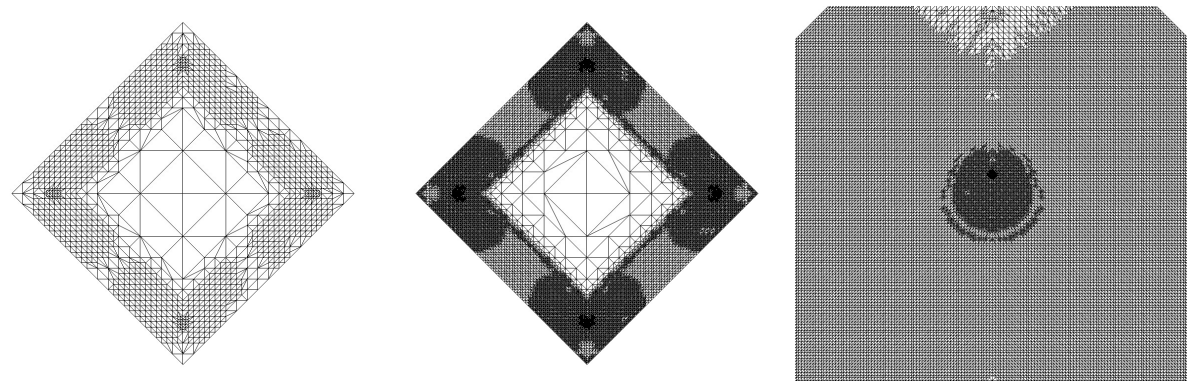

Figure 5. Adaptively refined triangulations $\mathcal{T}_{6}, \mathcal{T}_{9}$, and a zoom into the upper corner of $\mathcal{T}_{12}$.

As no exact solution is available, we cannot compare our estimator with the true error. Hence, Figure 4 just shows the hierarchical estimator $\eta_{j}^{2}$ and the extra term $\rho_{j}^{2}$ over the number of unknowns $n_{j}$. Let us first consider the left picture where the underlying sequence of grids is obtain by uniform refinement. Even though the solution is only in $H^{s}(\Omega)$ with $s<3 / 2$, we observe $\eta_{j}^{2}=\mathcal{O}\left(h_{j}^{2}\right)=\mathcal{O}\left(n_{j}^{-1}\right)$ which suggests optimal order of convergence. The reason is that the obstacle $\psi$, and thus the singularity of the solution along the diagonals, is exactly resolved by the grid. Note that the extra term $\rho_{j}^{2}$ is of higher order again and, in contrast to the first example, the exceptional nodes $\mathcal{N}_{j, b}$ are now concentrated along the approximate free boundary. In the right picture in Figure 4, the underlying grid is obtained by adaptive refinement. As expected, the hierarchical error estimator decays with the same (optimal) order as in the uniform case.

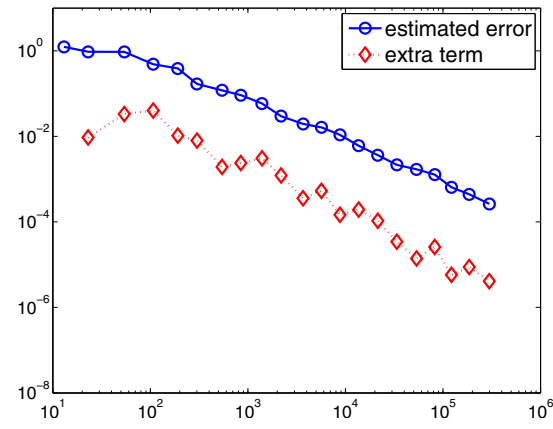

FiguRE 6 . The hierarchical estimator $\eta_{j}^{2}$ over the number of unknowns $n_{j}$ for the perturbed initial triangulation $\tilde{\mathcal{T}}_{1}$ not resolving the singularities of $\psi$ : Uniform refinement (left) and adaptive refinement (right).

The strong locality of the hierarchical error estimator is illustrated in Figure 5 showing $\mathcal{T}_{6}, \mathcal{T}_{9}$, and a zoom into the upper corner of $\mathcal{T}_{12}$. In contrast to related residual indicators 21], the hierarchical indicators do not produce any refinement within the coincidence set, where the obstacle $\psi$ and thus the solution is represented exactly. Instead, adaptive refinement concentrates at the free boundary, 

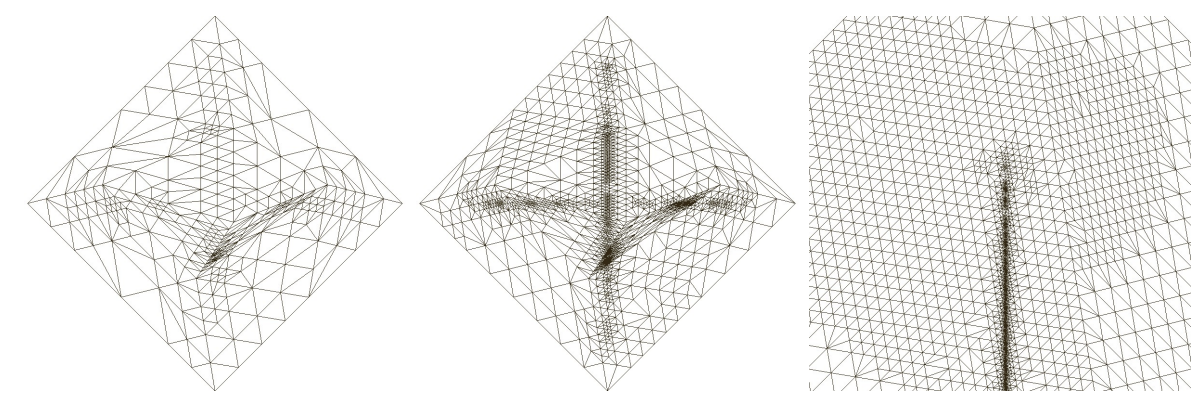

FIGURE 7. Adaptively refined triangulations $\tilde{\mathcal{T}}_{6}, \tilde{\mathcal{T}}_{9}$, and a zoom into the upper corner of $\tilde{\mathcal{T}}_{17}$ for the perturbed initial triangulation $\tilde{\mathcal{T}}_{0}$.

particularly at the cusps, which perfectly reflects the corresponding lack of regularity. According to Figure 4 , the gain from a reduced number of unknowns in the coincidence set is even compensated by overrefinement in the neighborhood of the singularities. In this example, the efficiency of the underlying adaptive refinement strategy could be improved by selecting a larger $\theta$ (marking fewer triangles for refinement), but this is not our interest here.

In order to study a more general situation, we now start from the initial triangulation $\tilde{\mathcal{T}}_{1}$ as obtained by connecting the midpoints of the edges of $\mathcal{T}_{0}$ and then moving the interior vertices away from the diagonals. As a consequence, the singularities of $\psi$ and thus of the solution inside of the coincidence set are no longer resolved. This immediately affects the evolution of the error estimator $\eta_{j}^{2}$ in course of refinement, as illustrated in Figure 6. In the left picture, associated with uniform refinement of $\tilde{\mathcal{T}}_{1}$, we now observe $\eta_{j}^{2}=\mathcal{O}\left(h_{j}\right)=\mathcal{O}\left(n_{j}^{-1 / 2}\right)$. This nicely reflects the reduced regularity of the continuous solution. It is interesting to see that adaptive refinement based on the hierarchical error indicators now preserves the optimal order $\eta_{j}^{2}=\mathcal{O}\left(n_{j}^{-1}\right)$. As shown in Figure 17, adaptive refinement no longer concentrates on the free boundary but on the dominating singularity along the diagonals. Theoretical justification of these observations will be the subject of future research.

\section{REFERENCES}

[1] M. Ainsworth and J.T. Oden. A Posteriori Error Estimation in Finite Element Analysis. John Wiley, New York, 2000. MR1885308 (2003b:65001)

[2] R.E. Bank and A. Weiser. Some a posteriori error estimators for elliptic partial differential equations. Math. Comp., 44:283-301, 1985. MR777265 (86g:65207)

[3] S. Bartels and C. Carstensen. Averaging techniques yield reliable a posteriori finite element error control for obstacle problems. Numer. Math., 99(2):225-249, 2004. MR2107431 (2005m:35067)

[4] F.A. Bornemann, B. Erdmann, and R. Kornhuber. A posteriori error estimates for elliptic problems in two and three space dimensions. SIAM J. Numer. Anal., 33:1188-1204, 1996. MR1393909 (98a:65161)

[5] D. Braess, R.H.W. Hoppe, and J. Schöberl. A posteriori estimators for obstacle problems by the hypercircle method. Comp. Visual. Sci., 11:351-362, 2008. MR2425501 (2009d:49010)

[6] F. Brezzi, W.W. Hager, and P.A. Raviart. Error estimates for the finite element solution of variational inequalities I. Numer. Math., 28:431-443, 1977. MR0448949 (56:7254)

[7] Z. Chen and R.H. Nochetto. Residual type a posteriori error estimates for elliptic obstacle problems. Numer. Math., 84:527-548, 2000. MR:1742264(2001c:65134) 
[8] P.G. Ciarlet. The Finite Element Method for Elliptic Problems. North-Holland, Amsterdam, 1980. MR608971 (82c:65068)

[9] B. Delaunay. Sur la sphère vide. Bull. Acad. Sci. URSS, 7:793-800, 1934.

[10] P. Deuflhard, P. Leinen, and H. Yserentant. Concepts of an adaptive hierarchical finite element code. IMPACT Comput. Sci. Engrg., 1:3-35, 1989.

[11] W. Dörfler. A convergent adaptive algorithm for Poisson's equation. SIAM J. Numer.Anal., 33:1106-1124, 1996. MR.1393904 (97e:65139)

[12] W. Dörfler and R.H. Nochetto. Small data oscillation implies the saturation assumption. Numer. Math., 91:1-12, 2002. MR.1896084(2003e:65195)

[13] C. Gräser, R. Kornhuber, and U. Sack. On hierarchical error estimators for time-discretized phase field models. To appear in the Proceedings of ENUMATH 2009.

[14] R.H.W. Hoppe and R. Kornhuber. Adaptive multilevel methods for obstacle problems. SIAM J. Numer. Anal., 31(2):301-323, 1994. MR1276702 (95c:65181)

[15] D. Kinderlehrer and G. Stampacchia. An Introduction to Variational Inequalities and Their Applications. Academic Press, New York, 1980. MR567696 (81g:49013)

[16] R. Kornhuber. A posteriori error estimates for elliptic variational inequalities. Computers Math. Applic., 31:49-60, 1996. MR.1385549 (97a:65091)

[17] R. Kornhuber. Adaptive Monotone Multigrid Methods for Nonlinear Variational Problems. Teubner, Stuttgart, 1997. MR.1469497 (98e:65054)

[18] S. Korotov and M. Kř́žžek. Global and local refinement techniques yielding nonobtuse tetrahedral partitions. Comput. Math. Applic., 50:1105-1113, 2005. MR2167747 (2006k:65330)

[19] S. Korotov, M. Kř́žzek, and P. Neittaanmäki. Weakened acute type condition for tetrahedral triangulations and the discrete maximum principle. Math. Comp., 70:107-119, 2000. MR1803125 (2001i:65126)

[20] W. Liu and N. Yan. A posteriori error estimators for a class of variational inequalities. J. Sci. Comput., 15:361-393, 2000. MR.1828748 (2002f:65165)

[21] R.H. Nochetto, K.G. Siebert, and A. Veeser. Pointwise a posteriori error control for elliptic obstacle problems. Numer. Math., 95:631-658, 2003. MR.1993943(2004g:49055)

[22] R.H. Nochetto, K.G. Siebert, and A. Veeser. Fully localized a posteriori error estimators and barrier sets for contact problems. SIAM J. Numer. Anal., 42:2118-2135, 2005. MR2139239 (2006g:65179)

[23] O. Sander. Multi-dimensional Coupling in a Human Knee Model. Ph.D. thesis, FU Berlin, 2008.

[24] K.G. Siebert and A. Veeser. A unilaterally constrained quadratic minimization with adaptive finite elements. SIAM J. Optim., 18:260-289, 2007. MR2299684(2008c:65347)

[25] A. Veeser. Efficient and reliable a posteriori error estimators for elliptic obstacle problems. SIAM J. Numer. Anal., 39:146-167, 2001. MR1860720 (2002g:65150)

[26] R. Verfürth. A Review of A Posteriori Error Estimation and Adaptive Mesh-Refinement Techniques. Wiley and Teubner, 1996.

[27] J. Xu. Theory of multilevel methods. Technical Report AM 48, Pennsylvania State University, Department of Mathematics, University Park, USA, 1989.

[28] J. Xu. Iterative methods by space decomposition and subspace correction. SIAM Review, 34:581-613, 1992. MR1193013 (93k:65029)

[29] J. Xu and L. Zikatanov. A monotone finite element scheme for convection diffusion equations. Math. Comp., 68:1429-1446, 1999. MR:1654022 (99m:65225)

[30] H. Yserentant. On the multilevel splitting of finite element spaces. Numer. Math., 49:379-412, 1986. MR853662(88d:65068a)

[31] H. Yserentant. Two preconditioners based on the multilevel splitting of finite element spaces. Numer. Math., 58:163-184, 1990. MR1069277 (91j:65076)

[32] O.C. Zienkiewicz, J.P. de S.R. Gago, and D.W. Kelly. The hierarchical concept in finite element analysis. Computers \& Structures, 16:53-65, 1983.

[33] Q. Zou, A. Veeser, R. Kornhuber, and C. Gräser. Hierarchical error estimates for the energy functional in obstacle problems. Technical Report 575, Matheon, 2009. 
Freie Universität Berlin, Institut für Mathematik, Arnimallee 6, D-14195 Berlin, GERMANY

E-mail address: kornhuber@math.fu-berlin.de

University Guangzhou, Department of Scientific Computation and Computer AppliCations, Guangzhou, 510275, People's Republic of China

E-mail address: mcszqs@mail.sysu.edu.cn 